\title{
Antibacterial effects of vancomycin in combination with methicillin against methicillin-resistant and methicillin- sensitive Staphylococcus aureus
}

\author{
Ali Abdolahi ${ }^{1}$, Alireza Khodavandi ${ }^{* *}$ \\ ${ }^{1}$ Department of Microbiology, Yasooj Branch, Islamic Azad University, Yasooj, Iran \\ ${ }^{2}$ Department of Biology, Gachsaran Branch, Islamic Azad University, Gachsaran, Iran
}

*Corresponding Author: Alireza Khodavandi, Department of Biology, Gachsaran Branch, Islamic Azad University, Gachsaran, Iran.Tel: 00987432332033, Email: alireza_khodavandi@yahoo.com,khodavandi@iaug.ac.ir

\begin{abstract}
Background and aims: Staphylococcus aureus is considered as a frequent cause of infections. Therefore, antibacterial agents including $\beta$-lactam are normally used to treat these infections while the emergence of antibiotic resistance is one of the major clinical problems in this respect. Accordingly, combination antibacterial therapy is one way to alleviate this problem. As a result, the present study aimed to investigate the combined effects of vancomycin and methicillin on clinical isolates of methicillin-resistant S. aureus (MRSA) and methicillinsensitive $S$. aureus (MSSA). In addition, a series of follow-up studies were performed to evaluate the antibacterial activity.

Methods: The current cross-sectional study was conducted on 40 hospitalized patients from various clinical samples such as pus/wound swabs, blood, urine and sputum during a 6-month period in 2017. To this end, the antibacterial activities of vancomycin and methicillin, alone and in combination were investigated against MRSA and MSSA. Eventually, the inhibitory effects of vancomycin and methicillin alone and in combination were studied on the growth profile of MRSA and MSSA, as well as the expression of mecA gene.

Results: Based on the results, the significant synergistic and partial synergistic activity with fractional inhibitory concentration (FIC) and fractional bactericidal concentration (FBC) indexes ranged from 0.27-0.938 and 0.313-0.844, respectively, in the combination of vancomycin and methicillin in MRSA and MSSA isolates. Further, the obtained data indicated that the combination of vancomycin and methicillin had a synergistic effect against MRSA isolates $(P<0.01)$. The expression levels of the mecA gene were down-regulated by 5.25fold in the combination of vancomycin and methicillin in MRSA isolate $(P<0.001)$.

Conclusion: In general, these events may reflect the potential uses of vancomycin and methicillin combination against MRSA. However, a greater understanding of the underlying molecular mechanisms of vancomycin and methicillin in combination could contribute to the development of therapeutic strategies.

Keywords: Combination Therapy; Methicillin-resistant Staphylococcus aureus; mecA
\end{abstract}

Received: 13 March 2018, Accepted: 21 July 2018, ePublished: 4 April 2019

\section{Introduction}

Staphylococcus aureus is a human commensal and a widespread cause of life-threatening systemic infections. The $\beta$-lactam antibiotics are regarded as the antibacterial drugs of choice for treating the $S$. aureus infections. In fact, their target mechanisms inhibit the synthesis of bacterial cell walls and thus lead to bacterial cell death $(1,2)$. In addition, methicillin-resistant $S$. aureus (MRSA) is the major hospital- and community-acquired pathogen that develops multiple drug resistance to $\beta$-lactam antibiotics. MRSA isolates appear as a common cause of the disease and death and impose severe economic problems (3-8).

Antibacterial resistance is due to the expression of a nonnative gene encoding a penicillin binding-protein (PBP2a), acquired from another species, with lower affinity for $\beta$-lactams. PBP2a remains active in the presence of typically inhibitory concentrations of $\beta$-lactam antibiotics thus allows cell wall biosynthesis. Further, PBP2a is encoded by the mecA gene localized in a unique segment of the DNA referred to as the staphylococcal chromosome cassette, which is transferred between the bacteria by horizontal gene transfer and the expression of which is controlled through a proteolytic signal transduction pathway $(9,10)$.

Vancomycin, a glycopeptide antibiotic originally isolated from Streptomyces orientalis, serves as the cornerstone of therapy against serious MRSA infections. The increased use of vancomycin has led to multiple exposures of MRSA isolates to this drug and the development of microbial resistance $(11,12)$. Several alternative agents to vancomycin have become available for the treatment of MRSA infections including linezolid, daptomycin, tigecycline, and quinupristin/dalfopristin. Moreover, the use of all these drugs is clinically limited due to the high cost and/or a substantial risk of adverse effects. Recent investigations in antibacterial development encompass using the combination therapy with two or more 
antibacterial compounds. However, several combined therapies with vancomycin are reported (13-18) as well. So far, a limited body of data has published regarding the efficacy of vancomycin in combination with methicillin on genes contributing to the antibacterial resistance of $S$. aureus. Therefore, the present study was designed to evaluate the combined effects of vancomycin and methicillin on clinical isolates of MRSA and methicillinsensitive $S$. aureus (MSSA) and conduct a series of followup studies in order to investigate their antibacterial activity.

\section{Materials and Methods}

Patients and samples

In this cross-sectional study which was implemented over a 6-month period in 2017, various clinical samples such as pus/wound swabs, blood, urine and sputum were randomly collected from 40 hospitalized patients, admitted to Shahid Beheshti Hospital of Yasooj, as well as Shahid Rajaee hospital of Gachsaran, and immediately transported to the laboratory. Then, the clinical isolates of $S$. aureus were identified based on their morphological, biochemical, and molecular characteristics such as colonies with staphylococcal morphology on blood agar (Merck, Darmstadt, Germany), Mannitol salt phenol red agar (Merck), Baird-Parker agar (Merck), gram staining, carbohydrate fermentation, catalase, slide/ tube coagulase, oxidase, DNase, urease and VP tests, along with bacitracin and novobiocin susceptibility tests. Next, molecular identification was performed using the specific 16S rRNA gene with specific primers Staph756F (5'AACTCTGTTATTAGGGAAGAACA3') and Staph750R (5'CCACCTTCCTCCGGTTTGTCACC3'). Furthermore, $S$. aureus isolates were stored at $-80^{\circ} \mathrm{C}$ in Trypticase soy broth (Merck, Germany) with 20\% glycerol. MSSA (ATCC 25923) was purchased from the Iranian Research Organization for Science and Technology. In addition, the antibacterial susceptibility was determined by the disk diffusion method, samples were transferred to the Clinical and Laboratory Standards Institute (CLSI) in order to detect MRSA. The antibiotic such as methicillin $(5 \mu \mathrm{g})$, oxacillin $(1 \mu \mathrm{g})$, gentamycin $(10 \mu \mathrm{g})$, vancomycin $(30 \mu \mathrm{g})$, cefixime $(5 \mu \mathrm{g})$, cephalexin $(30 \mu \mathrm{g})$, cefoxitin $(30 \mu \mathrm{g})$, and ciprofloxacin $(5 \mu \mathrm{g})$ were used, which were purchased from PADTAN TEB Company (Tehran, Iran). Finally, the results of the antibacterial susceptibility were analyzed using WHONET software 2017 (19-23).

The antibacterial susceptibility of vancomycin and methicillin alone and in combination against MRSA and MSSA

Antibacterial susceptibility was determined by the broth microdilution method as described in the CLSI M07-A10 (24) and CLSI supplement M100 (20). Briefly, $100 \mu \mathrm{L}$ of the two-fold dilution of different concentrations of vancomycin (within the range of 0.125-64 $\mu \mathrm{g} / \mathrm{mL}$ ) and methicillin (within the range of $0.125-64 \mu \mathrm{g} / \mathrm{mL}$ ), alone or in combination, were prepared in MuellerHinton broth (Merck) in the wells of 96-well U-bottom microtiter plates. These plates were then inoculated at $35^{\circ} \mathrm{C}$ with approximately $5 \times 10^{4}$ colony-forming units $(\mathrm{CFU}) / \mathrm{mL}$ of an overnight culture grown in MuellerHinton broth. After 24 hours of incubation, the minimal inhibitory concentrations (MICs) were measured at 630 nm using a Stat Fax 303 Reader (Awareness Technology, Inc., USA). The minimum bactericidal concentration (MBC) was determined by subculturing aliquots from MIC determination onto Mueller-Hinton agar plates. The MBC was defined as the lowest concentration of the antibacterial agents that killed at least $99.9 \%$ of the initial inoculums.

Moreover, drug interaction was defined as synergistic, additive, indifferent, or antagonistic based on the fractional inhibitory concentration (FIC) index employing the results of MICs. Additionally, the FIC index was calculated according to the following formulas:

FIC index $=$ (MIC of drug $\mathrm{x}$, in combination/MIC of drug $\mathrm{x}$, tested alone $)+(\mathrm{MIC}$ of drug $\mathrm{y}$, in combination/ MIC of drug y, tested alone), and

Fractional bactericidal concentration $(\mathrm{FBC})=(\mathrm{MBC}$ of drug $\mathrm{x}$, in combination/MBC of drug $\mathrm{x}$, tested alone) + (MBC of drug $y$, in combination/MBC of drug $y$, tested alone).

In addition, the FIC and FBC indexes were interpreted as follows: synergy, FIC $\leq 0.5$; partial synergy, FIC $>0.5$ while $<1.0$; additive effect $\mathrm{FIC}=1.0$; indifference, FIC $>1.0$ whereas <4.0; antagonism, FIC $\geq 4.0(25,26)$.

The inhibitory effect of vancomycin and methicillin alone and in combination on growth profile of MRSA and MSSA using the optical density

The growth profile was measured using the optical density in order to determine the inhibitory effect of the vancomycin and methicillin alone and in combination against MRSA and MSSA. The test was conducted utilizing the test tubes containing Mueller-Hinton broth, along with vancomycin and methicillin alone and in combination with $1 \times$ MIC concentration. The colony suspension (0.5 McFarland) was added to the tube. Then, the inhibitory effect of vancomycin and methicillin alone and in combination on the growth profile was measured using the spectrophotometer at $630 \mathrm{~nm}$ wavelength at different time intervals such as $0,4,8,12,16,20$, and 24 hours (27).

The expression analysis of mecA gene by real-time polymerase chain reaction

The expression of mecA gene in MRSA treated with vancomycin and methicillin alone and in combination 
was analyzed by quantitative real-time polymerase chain reaction (PCR). To this end, total RNA was extracted from the bacterial cells using the RNeasy Mini Kit (Qiagen, Hilden, Germany) according to the manufacturer's instructions. In addition, the RNA samples were treated with RNase-Free DNase Set (Qiagen) in order to eliminate the traces of genomic DNA. Further, the ratio of absorbance at A260/A280 and A260/A230 derived from the spectrophotometric NanoDrop ${ }^{\circledR}$ ND-1000 (NanoDrop Technologies Inc., Wilmington, DE) analysis was applied to assess the quantity and purity of the extracted RNA. Furthermore, the integrity of the total RNA was confirmed by agarose gel electrophoresis. Moreover, the total RNA $(0.5 \mu \mathrm{g})$ was reverse transcribed into single-stranded cDNA with Moloney-Murine leukemia virus reverse transcriptase and random hexamers (Fermentas, USA) based on the manufacturer's instructions. Additionally, $S$. aureus mecA gene was amplified from the synthesized cDNA with the primer as listed in Table 1. In addition, $\operatorname{gyr} B$ was established as a house-keeping gene in order to normalize the dissimilar RNA concentrations during the RNA extraction.

Real-time PCR experiments were performed on a Bio-Rad MiniOpticon ${ }^{\text {TM }}$ system (USA) using SYBR Green qPCR Master Mix (Fermentas, EU). PCR cycling conditions included an initial step at $50^{\circ} \mathrm{C}$ for 2 minutes, holding at $95^{\circ} \mathrm{C}$ for 8 minutes, 35 cycles of denaturation at $95^{\circ} \mathrm{C}$ for 10 seconds and annealing at $57^{\circ} \mathrm{C}$ for 15 seconds, and finally, the melting reaction at $72-78^{\circ} \mathrm{C}$. The relative quantitation of the gene expression was obtained by the Pfaffl method (29,30).

\section{Statistical analysis}

The results are expressed as mean value \pm standard error of three replicates. Moreover, all experiments were repeated three times in order to compensate for possible errors.

Table 1. Oligonucleotide primers used for PCR

\begin{tabular}{llc}
\hline Primer & Sequence & Reference \\
\hline MecA147-F & 5' GTG AAG ATA TAC CAA GTG ATT3' & $(28)$ \\
MecA147-R & 5' ATG CGC TAT AGA TTG AAA GGA T3' \\
gyrB-F & 5' GTC GAA GGG GAC TCT G 3' \\
gyrB- $R$ & 5' GCT CCA TCC ACA TCG G 3' & \\
\hline
\end{tabular}

Abbreviation: $\mathrm{PCR}$, polymerase chain reaction.
Additionally, the data were analyzed by the analysis of variance (ANOVA). In addition, the comparison of the two means was calculated using Tukey post hoc test. $P \leq$ 0.05 was considered statistically significant. Eventually, the statistical analysis was performed using the SPSS software, version 21 (SPSS Inc., Chicago, IL).

\section{Results}

Totally, 40 cases of $S$. aureus infections were collected from hospitalized patients during 6 months. Then, clinical isolates of the $S$. aureus were phenotypically identified and their reliability was confirmed by DNA sequencing. A number of $30(75 \%)$ out of 40 clinical isolates were documented as the MRSA while 10 (25\%) clinical isolates were identified as MSSA. Table 2 represents the clinical isolates of $S$. aureus based on their susceptibilities to antibiotics. A total of $45 \%$ of the clinical isolates of S. aureus have multidrug resistance patterns. In addition, $15 \%$ of these isolates are identified as possible extensively drug-resistant while no possible pandrug-resistant is detected.

The antibacterial activity of vancomycin and methicillin alone and in combination against the clinical isolates of $S$. aureus (i.e., MRSA and MSSA) were performed according to clinical and laboratory standards institute guidelines. Based on the results, the combination of vancomycin and methicillin led to a reduction in the MICs/MBCs regarding the clinical isolates of MRSA and MSSA. Further, vancomycin in combination with methicillin displayed $37.5 \%$ synergistic and partial synergistic effects with FIC and FBC indexes ranging from 0.270.938 and $0.313-0.844$, respectively. Furthermore, the combination effects of vancomycin with methicillin were additive (i.e., FIC and FBC indexes $=1$ ) against 10\% of the clinical isolates of MRSA and MSSA. The vancomycin in combination with methicillin was indifferent against $52.5 \%$ of MRSA and MSSA clinical isolates of by the FIC and $\mathrm{FBC}$ indexes within the ranges of 1.125-2.04 and $1.125-2.5$, respectively. Conversely, vancomycin alone was found to be active against all forty study clinical isolates with MICs between 0.5 and $2 \mu \mathrm{g} / \mathrm{mL}$ although methicillin alone revealed resistance against all the clinical isolates of S. aureus. Finally, the combination of vancomycin with

Table 2. The antibiotic susceptibilities of clinical isolates of Staphylococcus aureus in hospitalized patients

\begin{tabular}{|c|c|c|c|c|c|c|}
\hline Antibiotic Name & Code & Antibiotic Class & Antibiotic Subclass & R (\%) & I (\%) & S (\%) \\
\hline Cefixime & CFM_ND5 & Cephems-Oral & Cephalosporins & 35 & 37.5 & 27.5 \\
\hline Ciprofloxacin & CIP_ND5 & Quinolones & Fluoroquinolones & 45 & 27.5 & 27.5 \\
\hline Cefoxitin & FOX_ND30 & Cephems & Cephamycins & 75 & 20 & 5 \\
\hline Gentamicin & GEN_ND10 & Aminoglycosides & & 15 & 27.5 & 57.5 \\
\hline Cephalexin & LEX_ND30 & Cephems-Oral & Cephalosporins & 32.5 & 40 & 27.5 \\
\hline Methicillin & MET_ND5 & Penicillins & Penicillins (stable) & 100 & 0 & 0 \\
\hline Oxacillin & OXA_ND1 & Penicillins & Penicillins (stable) & 75 & 2.5 & 22.5 \\
\hline Vancomycin & VAN_ND30 & Glycopeptides & Glycopeptides & 0 & 0 & 100 \\
\hline
\end{tabular}


methicillin significantly reduced the MICs of methicillin against all the clinical isolates of $S$. aureus (Table 3 ).

The inhibitory effect of the vancomycin and methicillin alone and in combination against MRSA and MSSA are illustrated in Figure 1 with optical density as the $y$ axis and the incubation time as the $x$ axis. The growth profile of MRSA isolates are not inhibited by methicillin, instead, they continue to multiply, albeit at a lower rate and lower level of saturation compared to the untreated control while the vancomycin and methicillin in combination with synergistic effects inhibit the growth of MRSA isolates compared to the untreated control $(P<0.01)$. Moreover, as displayed in Figure 1C, no inhibitory effects were observed in MRSA isolates treated with the combination of vancomycin and methicillin with indifference effects.

Additionally, the results of relative quantitative realtime PCR showed that vancomycin and methicillin alone and in combination induced changes in the expression

Table 3. The MIC, MBC, FIC, and FBC values of vancomycin and methicillin alone and in combination against the clinical isolates of Staphylococcus aureus

\begin{tabular}{|c|c|c|c|c|c|c|c|c|}
\hline \multirow{2}{*}{ Isolates/Antibacterial } & \multicolumn{2}{|c|}{ Vancomycin } & \multicolumn{2}{|c|}{ Methicillin } & \multicolumn{3}{|c|}{ Vancomycin/Methicillin } & \multirow{2}{*}{ Outcome } \\
\hline & MIC & MBC & MIC & MBC & MIC & MBC & $\mathrm{FIC} / \mathrm{FBC}$ & \\
\hline S. aureus ATCC 25923 & 1 & 2 & 0.5 & 1 & 0.25 & 0.5 & $0.75 / 0.75$ & Partial synergy/Partial synergy \\
\hline $\mathrm{Cl}-1^{*}$ & 1 & 2 & 6 & 8 & 0.5 & 0.75 & $0.583 / 0.469$ & Synergy/Synergy \\
\hline $\mathrm{Cl}-2^{*}$ & 2 & 2 & 12 & 16 & 2 & 2 & $1.17 / 1.125$ & Indifference/Indifference \\
\hline $\mathrm{Cl}-3^{*}$ & 1 & 2 & 1 & 2 & 0.5 & 1 & $1.00 / 1.00$ & Additive/Additive \\
\hline $\mathrm{Cl}-4^{*}$ & 2 & 2 & 4 & 8 & 2 & 2 & $1.5 / 1.25$ & Indifference/Indifference \\
\hline $\mathrm{Cl}-5^{*}$ & 2 & 2 & 12 & 16 & 2 & 2 & $1.17 / 1.125$ & Indifference/Indifference \\
\hline $\mathrm{Cl}-6^{*}$ & 0.5 & 1 & 55 & 64 & 0.5 & 1 & $1.009 / 1.016$ & Additive/Additive \\
\hline $\mathrm{Cl}-7^{*}$ & 2 & 2 & 4 & 8 & 2 & 2 & $1.5 / 1.25$ & Indifference/Indifference \\
\hline $\mathrm{Cl}-8^{*}$ & 2 & 2 & 25 & 32 & 0.5 & 1 & $0.27 / 0.531$ & Synergy/Synergy \\
\hline $\mathrm{Cl}-9^{*}$ & 2 & 2 & 16 & 16 & 2 & 2 & $1.125 / 1.125$ & Indifference/Indifference \\
\hline $\mathrm{Cl}-10^{*}$ & 1 & 1 & 8 & 16 & 1 & 2 & $1.125 / 1.125$ & Indifference/Indifference \\
\hline $\mathrm{Cl}-11^{*}$ & 1 & 1 & 4 & 8 & 1 & 2 & $1.25 / 1.25$ & Indifference/Indifference \\
\hline $\mathrm{Cl}-12^{*}$ & 1 & 1 & 4 & 8 & 1 & 2 & $1.25 / 1.25$ & Indifference/Indifference \\
\hline $\mathrm{Cl}-13^{*}$ & 2 & 2 & 4 & 8 & 1 & 1 & $0.75 / 0.625$ & Partial synergy/Partial synergy \\
\hline $\mathrm{Cl}-14^{*}$ & 2 & 2 & 6 & 8 & 2 & 2 & $1.33 / 1.25$ & Indifference/Indifference \\
\hline $\mathrm{Cl}-15^{*}$ & 1 & 2 & 4 & 8 & 0.5 & 1 & $0.625 / 0.625$ & Partial synergy/Partial synergy \\
\hline $\mathrm{Cl}-16^{*}$ & 1 & 1 & 55 & 64 & 2 & 2 & $2.04 / 2.03$ & Indifference/Indifference \\
\hline $\mathrm{Cl}-17^{*}$ & 1 & 2 & 4 & 8 & 0.5 & 1 & $0.625 / 0.625$ & Partial synergy/Partial synergy \\
\hline $\mathrm{Cl}-18^{*}$ & 2 & 2 & 6 & 8 & 2 & 4 & $1.33 / 2.5$ & Indifference/Indifference \\
\hline $\mathrm{Cl}-19^{*}$ & 1 & 1 & 4 & 8 & 0.75 & 0.75 & $0.938 / 0.844$ & Partial synergy/Partial synergy \\
\hline $\mathrm{Cl}-20^{*}$ & 1 & 1 & 55 & 64 & 0.5 & 0.5 & $0.509 / 0.508$ & Synergy/Synergy \\
\hline $\mathrm{Cl}-21^{*}$ & 1 & 1 & 4 & 8 & 1 & 2 & $1.25 / 2.25$ & Indifference/Indifference \\
\hline $\mathrm{Cl}-22^{*}$ & 0.5 & 1 & 4 & 8 & 0.5 & 1 & $1.125 / 1.125$ & Indifference/Indifference \\
\hline $\mathrm{Cl}-23^{*}$ & 2 & 2 & 4 & 8 & 2 & 2 & $1.5 / 1.25$ & Indifference/Indifference \\
\hline $\mathrm{Cl}-24^{*}$ & 2 & 2 & 4 & 8 & 2 & 2 & $1.5 / 1.25$ & Indifference/Indifference \\
\hline $\mathrm{Cl}-25^{*}$ & 2 & 2 & 6 & 8 & 2 & 2 & $1.33 / 1.25$ & Indifference/Indifference \\
\hline $\mathrm{Cl}-26^{*}$ & 2 & 2 & 4 & 8 & 2 & 2 & $1.5 / 1.25$ & Indifference/Indifference \\
\hline $\mathrm{Cl}-27^{*}$ & 1 & 1 & 4 & 8 & 1 & 2 & $1.25 / 1.25$ & Indifference/Indifference \\
\hline $\mathrm{Cl}-28^{*}$ & 1 & 2 & 1 & 2 & 0.5 & 1 & $1.00 / 1.00$ & Additive/Additive \\
\hline $\mathrm{Cl}-29^{*}$ & 1 & 2 & 4 & 8 & 0.5 & 1 & $0.625 / 0.625$ & Partial synergy/Partial synergy \\
\hline $\mathrm{Cl}-30^{*}$ & 1 & 2 & 4 & 8 & 0.5 & 1 & $0.625 / 0.625$ & Partial synergy/Partial synergy \\
\hline $\mathrm{Cl}-31^{* *}$ & 0.5 & 1 & 4 & 8 & 0.5 & 1 & $1.125 / 1.125$ & Indifference/Indifference \\
\hline $\mathrm{Cl}-32^{* *}$ & 1 & 2 & 4 & 8 & 0.5 & 1 & $0.625 / 0.625$ & Partial synergy/Partial synergy \\
\hline $\mathrm{Cl}-33^{* *}$ & 1 & 1 & 4 & 8 & 1 & 1 & $1.25 / 1.125$ & Indifference/Indifference \\
\hline $\mathrm{Cl}-34^{* *}$ & 1 & 1 & 4 & 8 & 1 & 2 & $1.25 / 2.25$ & Indifference/Indifference \\
\hline $\mathrm{Cl}-35^{* *}$ & 1 & 1 & 4 & 8 & 0.5 & 0.75 & $0.625 / 0.844$ & Partial synergy/Partial synergy \\
\hline $\mathrm{Cl}-36^{* *}$ & 1 & 2 & 1 & 2 & 0.5 & 1 & $1.00 / 1.00$ & Additive/Additive \\
\hline $\mathrm{Cl}-37^{* *}$ & 2 & 2 & 6 & 8 & 0.5 & 0.5 & $0.333 / 0.313$ & Synergy/Synergy \\
\hline $\mathrm{Cl}-38^{* *}$ & 1 & 1 & 4 & 8 & 0.5 & 0.75 & $0.625 / 0.844$ & Partial synergy/Partial synergy \\
\hline $\mathrm{Cl}-39^{* *}$ & 2 & 2 & 8 & 16 & 0.5 & 1 & $0.313 / 0.563$ & Synergy/Synergy \\
\hline $\mathrm{Cl}-40^{* *}$ & 2 & 2 & 4 & 8 & 0.5 & 0.5 & $0.375 / 0.313$ & Synergy/Synergy \\
\hline
\end{tabular}

Abbreviations: $\mathrm{Cl}$, clinical isolates of the $S$. aureus (i.e., MRSA and MSSA); MIC, minimal inhibitory concentrations; $\mathrm{MBC}$, minimum bactericidal concentration; FIC, fractional inhibitory concentration; FBC, fractional bactericidal concentration.

* MRSA; ** MSSA. 
(A)

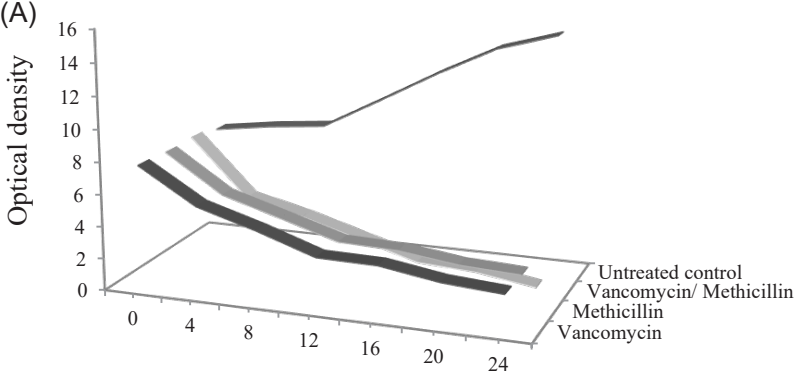

Incubation Time (h)

(B)
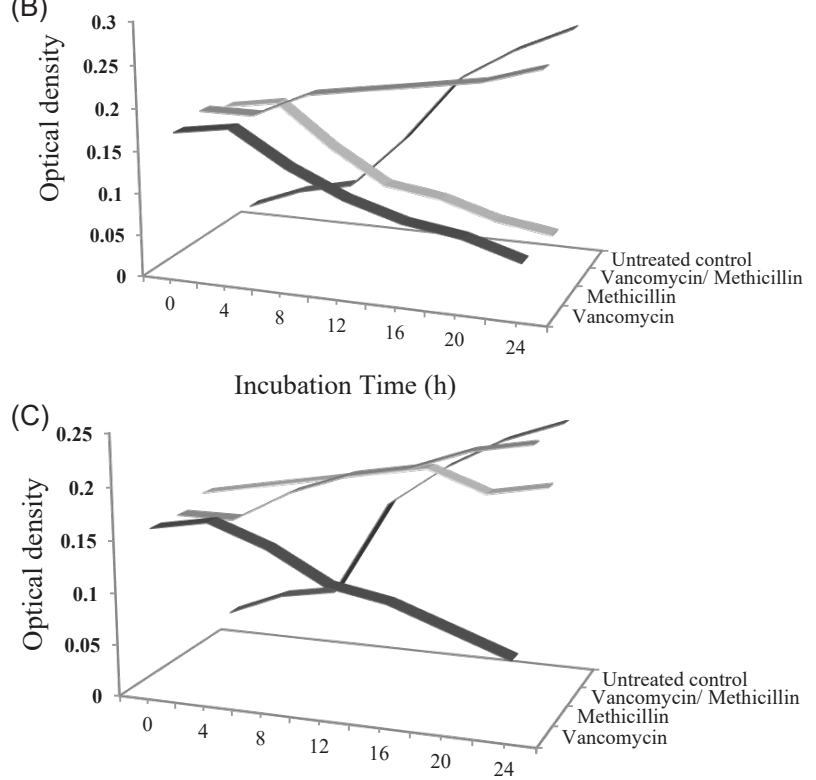

Incubation Time (h)

Figure 1. The inhibitory effect of vancomycin and methicillin alone and in combination on the growth profile of $S$. aureus. Note. A: $S$. aureus ATCC 25923 (MSSA); B: Cl-8; C: Cl-16.

levels of mecA gene in MRSA isolates with synergistic and indifference effects. However, the expression levels of mecA demonstrated no significant change in MRSA isolates treated with the vancomycin alone $(P=0.20)$. In addition, the expression of mec $A$ failed to induce any changes in MRSA treated with methicillin alone compared to the untreated control group $(P=0.38)$. Contrarily, the combination of vancomycin and methicillin significantly down-regulated the expression levels of mecA by 5.25fold in MRSA isolate with synergistic effect $(P<0.001)$. Moreover, there were no significant changes in the expression level of mec $A$ in MRSA isolate with indifference effect treated with the combination of vancomycin and methicillin (Figure 2). Ultimately, the analysis of the nucleotide sequence of the PCR products revealed a high similarity by the nucleotide BLAST in National Center for Biotechnology Information.

\section{Discussion}

Staphylococcus aureus is believed to be the most common human pathogen, leading to a wide range of infections.

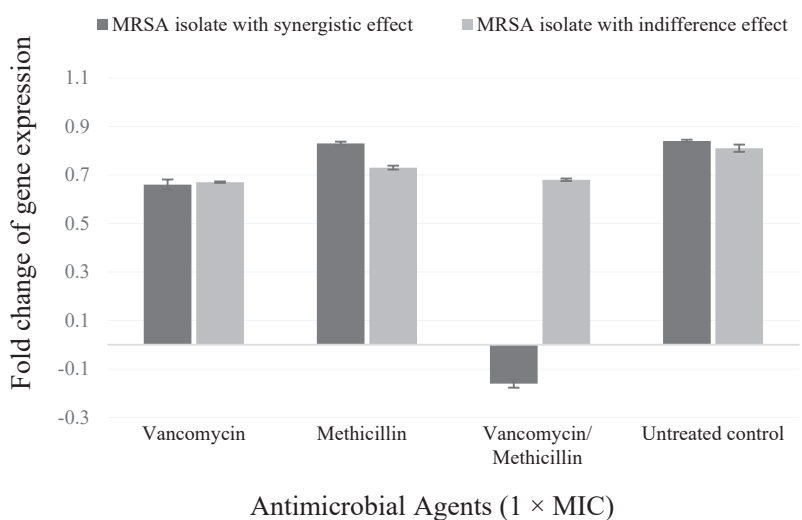

Figure 2. The relative quantitation of the gene expression of mecA in MRSA isolates treated with vancomycin and methicillin alone and in combination at concentrations of $1 \times$ MIC after 24 hours.

Note. Data are means \pm standard error of fold changes from three independent experiments amplified in triplicates.

Further, it expresses many virulence factors that contribute to diseases. The important virulence factors involved in the disease process can be divided into cell surface factors which recognize the adhesive matrix molecules (e.g., protein A, along with fibronectin-binding, collagenbinding, and clumping factor proteins), capsular polysaccharides, and staphyloxanthin, as well as secreted factors (e.g., superantigens, cytolytic toxins, and various exoenzymes and miscellaneous proteins) that play active roles in disarm host immunity (1,31-33).

According to previous studies $(1,2,8), \quad \beta$-lactam antibiotics demonstrate the most optimal treatment for $S$. aureus infections and lead to the prominent cause of resistance such as MRSA. Furthermore, the mechanisms of antibiotic activity of the methicillin against $S$. aureus possibly act by the inhibitory effects of the PBPs that are involved in the synthesis of peptidoglycan, causing cell wall synthesis and thus halting cell growth (34). Moreover, vancomycin has an important role in antibacterial activity and directly binds to the $\mathrm{C}$-terminal $\mathrm{D}$-Ala-D-Ala residues of lipid II, which prevents using the precursor for the cell wall synthesis (35).

The present study investigated the interactions between vancomycin and methicillin on the inhibition of MRSA and MSSA. Methicillin alone revealed resistance against all the clinical isolates of MRSA while the obtained data represented the significant activity of vancomycin alone against all the clinical isolates of MRSA and MSSA. Interestingly, the in vitro combination of vancomycin and methicillin displayed synergistic effects against MRSA and MSSA isolates. In recent years, many studies have reported the expanded use of combined antibacterial therapy with vancomycin for treating various infections of MRSA (3638). For example, Sy et al (39) showed the synergistic effect of the combination of vancomycin and $\beta$-lactams against the MRSA. Additionally, Tong et al (40) revealed the synergy between vancomycin or daptomycin and an 
anti-staphylococcal $\beta$-lactam antibiotic.

The findings of the current study indicated that the expression levels of the mecA gene were down-regulated in MRSA isolates with synergistic effect treated with the combination of vancomycin and methicillin. Conversely, the expression of mecA failed to demonstrate any changes in MRSA with indifference effect treated with the combination of vancomycin and methicillin. The results further confirm the possibility of changes in the mec $A$ gene (i.e., "see-saw effect") in MRSA with the synergistic effect in the combination of vancomycin and methicillin. However, there are limited studies regarding the combination therapy for the treatment of persistent MSSA bacteremia (14-18). The mechanism of synergy between vancomycin and betalactams against the MRSA is not well-understood and is unexpected given the inherent resistance of the MRSA to beta-lactams. Molecular base studies suggest vancomycin resistance-mediated re-sensitization to $\beta$-lactams (i.e., "see-saw effect") in MRSA in which the MRSA with rising vancomycin the MICs are associated with decreasing MICs to methicillin, possibly due to the changes in PBP or variations in the mecA gene $(17,41)$. Yang et al. (42) indicated that daptomycin-oxacillin combination was effective in reducing 3- to 4-fold in the oxacillin MIC and identified a daptomycin-oxacillin "see-saw" phenomenon in vitro. In addition, they reported the influence of the daptomycin-oxacillin combination in treating the experimental endocarditis caused by the MRSA evolved the "see-saw effect".

\section{Conclusion}

In general, these events may reflect the potential of the synergistic interaction of vancomycin and methicillin on MRSA. Further, the positive correlation between the synergism of the combination, the inhibitory effect of combination, and the expression of mecA gene may be due to a decrease in MRSA virulence. However, greater knowledge of molecular mechanisms of vancomycin and methicillin in combination could help develop the therapeutic strategies.

Conflict of interests

None.

Ethical considerations

This study was approved by the Research Ethics Committee of our institute (Ethical code 1180680). Further, the study protocol conformed to the ethical guidelines of the 2008 Declaration of Helsinki. Furthermore, written informed consent was obtained from the patients for utilizing the samples in the research.

\section{Acknowledgments}

The authors are thankful to the Islamic Azad University of Yasooj for financial support. Results of the current study are part of the MSc thesis $(1180680,95.02 .25)$

\section{References}

1. Klein E, Smith DL, Laxminarayan R. Hospitalizations and deaths caused by methicillin-resistant Staphylococcus aureus, United States, 1999-2005. Emerg Infect Dis. 2007;13(12):1840-6. doi: 10.3201/eid1312.070629.

2. Kohanski MA, Dwyer DJ, Collins JJ. How antibiotics kill bacteria: from targets to networks. Nat Rev Microbiol. 2010;8(6):423-35. doi: 10.1038/nrmicro2333.

3. Davies J, Davies D. Origins and evolution of antibiotic resistance. Microbiol Mol Biol Rev. 2010;74(3):417-33. doi: 10.1128/mmbr.00016-10.

4. Batabyal B, Kundu GKR, Biswas S. Methicillin-resistant Staphylococcus aureus: a brief review. Int Res J Biol Sci. 2012;1(7):65-71.

5. Klein EY, Sun L, Smith DL, Laxminarayan R. The changing epidemiology of methicillin-resistant Staphylococcus aureus in the United States: a national observational study. Am J Epidemiol. 2013;177(7):666-74. doi: 10.1093/aje/kws273.

6. Stryjewski ME, Corey GR. Methicillin-resistant Staphylococcus aureus: an evolving pathogen. Clin Infect Dis. 2014;58 Suppl 1:S10-9. doi: 10.1093/cid/cit613.

7. Uhlemann AC, Otto M, Lowy FD, DeLeo FR. Evolution of community- and healthcare-associated methicillin-resistant Staphylococcus aureus. Infect Genet Evol. 2014;21:563-74. doi: 10.1016/j.meegid.2013.04.030.

8. Damavandi MS, Safarpour Dehkordi M, Dehghan A, Heibati F, Taghaddosi R, Gholipour A. Detection of antiseptic resistance genes among Staphylococcus aureus colonising nurses and coagulase-negative staphylococci isolated from clinical specimens at teaching hospitals in southwest of Iran. Jundishapur J Microbiol. 2017;10(1):e39285. doi: 10.5812/ jjm.39285.

9. Llarrull LI, Fisher JF, Mobashery S. Molecular basis and phenotype of methicillin resistance in Staphylococcus aureus and insights into new beta-lactams that meet the challenge. Antimicrob Agents Chemother. 2009;53(10):4051-63. doi: 10.1128/aac.00084-09.

10. Peacock SJ, Paterson GK. Mechanisms of methicillin resistance in Staphylococcus aureus. Annu Rev Biochem. 2015;84:577601. doi: 10.1146/annurev-biochem-060614-034516.

11. Levine DP. Vancomycin: a history. Clin Infect Dis. 2006;42 Suppl 1:S5-12. doi: 10.1086/491709.

12. Rivera AM, Boucher HW. Current concepts in antimicrobial therapy against select gram-positive organisms: methicillinresistant Staphylococcus aureus, penicillin-resistant pneumococci, and vancomycin-resistant enterococci. Mayo Clin Proc. 2011;86(12):1230-43. doi: 10.4065/ mcp.2011.0514.

13. Micek ST. Alternatives to vancomycin for the treatment of methicillin-resistant Staphylococcus aureus infections. Clin Infect Dis. 2007;45 Suppl 3:S184-90. doi: 10.1086/519471.

14. Silva LV, Araujo MT, Santos KR, Nunes AP. Evaluation of the synergistic potential of vancomycin combined with other antimicrobial agents against methicillinresistant Staphylococcus aureus and coagulase-negative Staphylococcus spp strains. Mem Inst Oswaldo Cruz. 2011;106(1):44-50.

15. Dilworth TJ, Ibrahim O, Hall P, Sliwinski J, Walraven C, Mercier RC. beta-Lactams enhance vancomycin activity against methicillin-resistant Staphylococcus aureus bacteremia compared to vancomycin alone. Antimicrob Agents Chemother. 2014;58(1):102-9. doi: 10.1128/aac.01204-13.

16. Davis JS, Sud A, O'Sullivan MVN, Robinson JO, Ferguson PE, Foo $\mathrm{H}$, et al. Combination of vancomycin and beta-lactam therapy for methicillin-resistant Staphylococcus aureus bacteremia: a pilot multicenter randomized controlled trial. Clin Infect Dis. 2016;62(2):173-80. doi: 10.1093/cid/civ808.

17. Bartash R, Nori P. Beta-lactam combination therapy for the treatment of Staphylococcus aureus and Enterococcus species bacteremia: a summary and appraisal of the evidence. Int J 
Infect Dis. 2017;63:7-12. doi: 10.1016/j.ijid.2017.07.019.

18. Truong J, Veillette JJ, Forland SC. Outcomes of vancomycin plus a beta-lactam versus vancomycin only for treatment of methicillin-resistant Staphylococcus aureus bacteremia. Antimicrob Agents Chemother. 2018;62(2). doi: 10.1128/ aac.01554-17.

19. Clinical and Laboratory Standards Institute (CLSI). Performance standards for antibacterial disk susceptibility tests. Pennsylvania: Clinical and Laboratory Standards; 2015.

20. Clinical and Laboratory Standards Institute (CLSI). Performance standards for antibacterial susceptibility testing. Pennsylvania: Clinical and Laboratory Standards; 2017.

21. McClure JA, Conly JM, Lau V, Elsayed S, Louie T, Hutchins W, et al. Novel multiplex PCR assay for detection of the staphylococcal virulence marker Panton-Valentine leukocidin genes and simultaneous discrimination of methicillinsusceptible from -resistant staphylococci. J Clin Microbiol. 2006;44(3):1141-4. doi: 10.1128/jcm.44.3.1141-1144.2006.

22. Amini R, Abdulamir AS, Ling BP, Jahanshiri F, Hematian A, Zargar $M$, et al. Isolation and identification of methicillin resistant Staphylococcus aureus from keys of college students using different detection methods. Br Biotechnol J. 2012;2(1):13-25.

23. Juayang AC, de Los Reyes GB, de la Rama AJ, Gallega CT. Antibiotic resistance profiling of Staphylococcus aureus Isolated from clinical specimens in a tertiary hospital from 2010 to 2012. Interdiscip Perspect Infect Dis. 2014;2014:898457. doi: 10.1155/2014/898457.

24. Clinical and Laboratory Standards Institute (CLSI). Methods for dilution antibacterial susceptibility tests for bacteria that grow aerobically. Pennsylvania: CLSI; 2015.

25. Khodavandi A, Alizadeh F, Aala F, Sekawi Z, Chong PP. In vitro investigation of antifungal activity of allicin alone and in combination with azoles against Candida species. Mycopathologia. 2010;169(4):287-95. doi: 10.1007/s11046009-9251-3.

26. Lee YS, Jung EK, Cha JD. Synergistic effect between baicalein and antibiotics against clinic methicillin and vancomycinresistant Staphylococcus aureus. Chemotherapy. 2015;4(1):211. doi: 10.4172/2167-7700.1000141.

27. Low CF, Chong PP, Yong PV, Lim CS, Ahmad Z, Othman F. Inhibition of hyphae formation and SIR2 expression in Candida albicans treated with fresh Allium sativum (garlic) extract. J Appl Microbiol. 2008;105(6):2169-77. doi: 10.1111/j.13652672.2008.03912.x.

28. Zhang K, McClure JA, Elsayed S, Louie T, Conly JM. Novel multiplex PCR assay for characterization and concomitant subtyping of staphylococcal cassette chromosome mec types I to $\mathrm{V}$ in methicillin-resistant Staphylococcus aureus. J Clin Microbiol. 2005;43(10):5026-33. doi: 10.1128/ jcm.43.10.5026-5033.2005.

29. Sihto HM, Tasara T, Stephan R, Johler S. Validation of reference genes for normalization of qPCR mRNA expression levels in Staphylococcus aureus exposed to osmotic and lactic acid stress conditions encountered during food production and preservation. FEMS Microbiol Lett. 2014;356(1):134-40. doi: 10.1111/1574-6968.12491.

30. Khodavandi A, Harmal NS, Alizadeh F, Scully OJ, Sidik SM, Othman F, et al. Comparison between allicin and fluconazole in Candida albicans biofilm inhibition and in suppression of
HWP1 gene expression. Phytomedicine. 2011;19(1):56-63. doi: 10.1016/j.phymed.2011.08.060.

31. Costa AR, Batistao DWF, Ribas RM, Sousa AM, Pereira MO, Botelho CM. Staphylococcus aureus virulence factors and disease. In: Mendez-Vilas A, ed. Microbial pathogens and strategies for combating them: science, technology and education. Spain: Formatex; 2013:702-10.

32. Kobayashi SD, Malachowa N, DeLeo FR. Pathogenesis of Staphylococcus aureus abscesses. Am J Pathol. 2015;185(6):1518-27. doi: 10.1016/j.ajpath.2014.11.030.

33. Abbasi S, Khaledi M, Gholipour A, Heidari R. Assessment of the prevalence of Staphylococcus aureus in nose of the surgical staff of Hajar and Kashani's hospital in 2015. Journal of Shahrekord University of Medical Sciences. 2017;19(2):15. [Persian].

34. Reed P, Atilano ML, Alves R, Hoiczyk E, Sher X, Reichmann NT, et al. Staphylococcus aureus survives with a minimal peptidoglycan synthesis machine but sacrifices virulence and antibiotic resistance. PLoS Pathog. 2015;11(5):e1004891. doi: 10.1371/journal.ppat.1004891.

35. Hu Q, Peng H, Rao X. Molecular events for promotion of vancomycin resistance in vancomycin intermediate Staphylococcus aureus. Front Microbiol. 2016;7:1601. doi: 10.3389/fmicb.2016.01601.

36. Deresinski S. Vancomycin in combination with other antibiotics for the treatment of serious methicillinresistant Staphylococcus aureus infections. Clin Infect Dis. 2009;49(7):1072-9. doi: 10.1086/605572.

37. Kobayashi Y. Study of the synergism between carbapenems and vancomycin or teicoplanin against MRSA, focusing on S-4661, a carbapenem newly developed in Japan. J Infect Chemother. 2005;11(5):259-61. doi: 10.1007/s10156-0050402-2.

38. Tabuchi F, Matsumoto Y, Ishii M, Tatsuno K, Okazaki M, Sato $\mathrm{T}$, et al. Synergistic effects of vancomycin and beta-lactams against vancomycin highly resistant Staphylococcus aureus. J Antibiot (Tokyo). 2017;70(6):771-4. doi: 10.1038/ja.2017.7.

39. Sy CL, Huang TS, Chen CS, Chen YS, Tsai HC, Wann SR, et al. Synergy of beta-lactams with vancomycin against methicillinresistant Staphylococcus aureus: correlation of disk diffusion and checkerboard methods. J Clin Microbiol. 2016;54(3):5658. doi: 10.1128/jcm.01779-15.

40. Tong SY, Nelson J, Paterson DL, Fowler VG Jr, Howden BP, Cheng AC, et al. CAMERA2 - combination antibiotic therapy for methicillin-resistant Staphylococcus aureus infection: study protocol for a randomised controlled trial. Trials. 2016;17:170. doi: 10.1186/s13063-016-1295-3.

41. Renzoni A, Kelley WL, Rosato RR, Martinez MP, Roch M, Fatouraei $\mathrm{M}$, et al. Molecular bases determining daptomycin resistance-mediated resensitization to beta-lactams (seesaw effect) in methicillin-resistant Staphylococcus aureus. Antimicrob Agents Chemother. 2017;61(1):e01634-16. doi: 10.1128/aac.01634-16.

42. Yang SJ, Xiong YQ, Boyle-Vavra S, Daum R, Jones T, Bayer AS. Daptomycin-oxacillin combinations in treatment of experimental endocarditis caused by daptomycinnonsusceptible strains of methicillin-resistant Staphylococcus aureus with evolving oxacillin susceptibility (the "seesaw effect"). Antimicrob Agents Chemother. 2010;54(8):3161-9. doi: 10.1128/aac.00487-10. 